\title{
Applying Wavelet Entropy Principle in Fault Classification
}

\author{
S. El Safty, and A. El-Zonkoly
}

\begin{abstract}
The ability to detect and classify the type of fault plays a great role in the protection of power system. This procedure is required to be precise with no time consumption. In this paper detection of fault type has been implemented using wavelet analysis together with wavelet entropy principle. The simulation of power system is carried out using PSCAD/EMTDC. Different types of faults were studied obtaining various current waveforms. These current waveforms were decomposed using wavelet analysis into different approximation and details. The wavelet entropy of such decompositions is analyzed reaching a successful methodology for fault classification. The suggested approach is tested using different fault types and proven successful identification for the type of fault.
\end{abstract}

Keywords-Fault classification, wavelet transform, wavelet entropy.

\section{INTRODUCTION}

$T_{8}$ HE importance of protection of power system plays a great role in the successful operation of power system. The rapid and successful decision for tripping of circuit breaker has been focused on for a long time. The transient waveforms of current and voltage resulting during the fault has been thoroughly discussed. Due to the novel methods for signal conditioning, different methods were used in analyzing such waveforms. Many researchers used neural networks in identification of type of faults [1] others used wavelet analysis for fault detection and identification [2]. The results of analyzing fault signal using wavelet transformation method contain many useful data. Based on such transformation, the wavelet energy entropy in association with nerve fuzzy inference system is used for fault classification [3]. The wavelet entropy principle has been used in different applications in power system [4], [5].

In [6], wavelet entropy when used in feature pickup, neural network and fuzzy system were used to identify the fault. Wavelet transform generated time-frequency parameters and wavelet entropy generated characteristic vector. These characteristics were put into neural network to detect the fault transient then a fuzzy system was used to identify the fault.

In this paper, a much simpler algorithm is introduced to detect and identify the transmission line fault and also

S. El Safty, Associate professor, Electrical and computer control Department, College of Engineering, Arab Academy of Science and Technology, Alexandria, Egypt.

A. El-Zonkoly, lecturer, Electrical and computer control Department, College of Engineering, Arab Academy of Science and Technology, Alexandria, Egypt. determine the phases involved in the fault.

In this paper a novel algorithm is presented to detect the fault and identify its type using wavelet entropy energy. The main principle of wavelet entropy is first presented. Then algorithm used in fault detection and identification is discussed. Simulation of the proposed system in order to obtain different current signals for testing of the proposed technique has been done using PSCAD program. At the end, results for testing of the proposed technique are presented.

\section{PRINCIPLES OF WAVELET ENTROPY}

Transient signals have some characteristics such as high frequency and instant break. Wavelet transform is capable of revealing aspects of data that other signal analysis techniques miss and it satisfies the analysis need of electric transient signals. Usually, wavelet transform of transient signal is expressed by multi-revolution decomposition fast algorithm which utilizes the orthogonal wavelet bases to decompose the signal to components under different scales. It is equal to recursively filtering the signal with a high-pass and low-pass filter pair. The approximations are the high-scale, lowfrequency components of the signal produced by filtering the signal by a low-pass filter. The details are the low-scale, highfrequency components of the signal produced by filtering the signal by a high-pass filter. The band width of these two filters is equal. After each level of decomposition, the sampling frequency is reduced by half. Then recursively decompose the low-pass filter outputs (approximations) to produce the components of the next stage [6], [7].

Given a discrete signal $\mathrm{x}(\mathrm{n})$, being fast transformed at instant $\mathrm{k}$ and scale $\mathrm{j}$, it has a high-frequency component coefficient $\mathrm{D}_{\mathrm{j}}(\mathrm{k})$ and a low-frequency component coefficient $A_{j}(k)$. The frequency band of the information contained in signal components $D_{j}(k)$ and $A_{j}(k)$, obtained by reconstruction are as follows [5].

$$
\left\{\begin{array}{l}
D_{j}(k):\left[2^{-(j+1)} f_{s}, 2^{-j} f_{s}\right] \\
A_{j}(k):\left[0,2^{-(j+1)} f_{s}\right]
\end{array}(j=1,2, \ldots, m)\right.
$$

Where, $f_{s}$ is the sampling frequency.

The original signal sequence $x(n)$ can be represented by the sum of all components as follows [5].

$$
\begin{aligned}
x(n) & =D_{1}(n)+A_{1}(n)=D_{1}(n)+D_{2}(n)+A_{2}(n) \\
& =\sum_{j=1}^{J} D_{j}(n)+A_{J}(n)
\end{aligned}
$$


Various wavelet entropy measures were defined in [x1]. In this paper, the nonnormalized Shannon entropy will be used. The definition of nonnormalized Shannon entropy is as follows [8].

$$
E_{j}=-\sum_{k} E_{j k} \log E_{j k}
$$

Where $E_{j k}$ is the wavelet energy spectrum at scale $j$ and instant $\mathrm{k}$ and it is defined as follows.

$$
E_{j k}=\left|D_{j}(k)\right|^{2}
$$

\section{Proposed Algorithm}

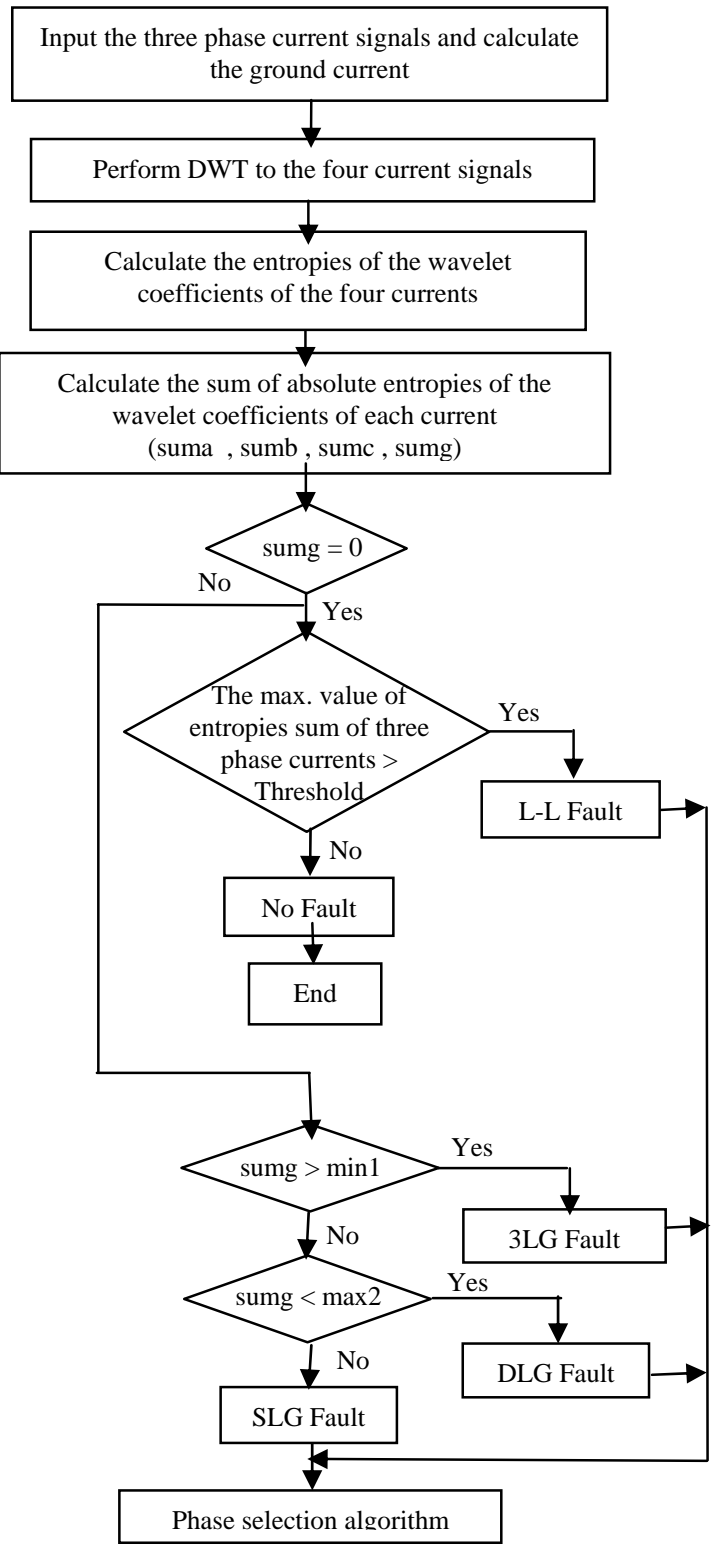

Fig. 1 Flow chart of fault detection and identification algorithm

\section{SYSTEM SIMULATION}

The system simulated is composed of two generators with transmission line between them. The PSCAD program is used for system simulation. Different types of faults at different locations along the transmission line are performed. The obtained results are used for implementation of the proposed technique and then for testing its validity.

Fig. 3 shows the current waveform for single line to ground fault at phase A.

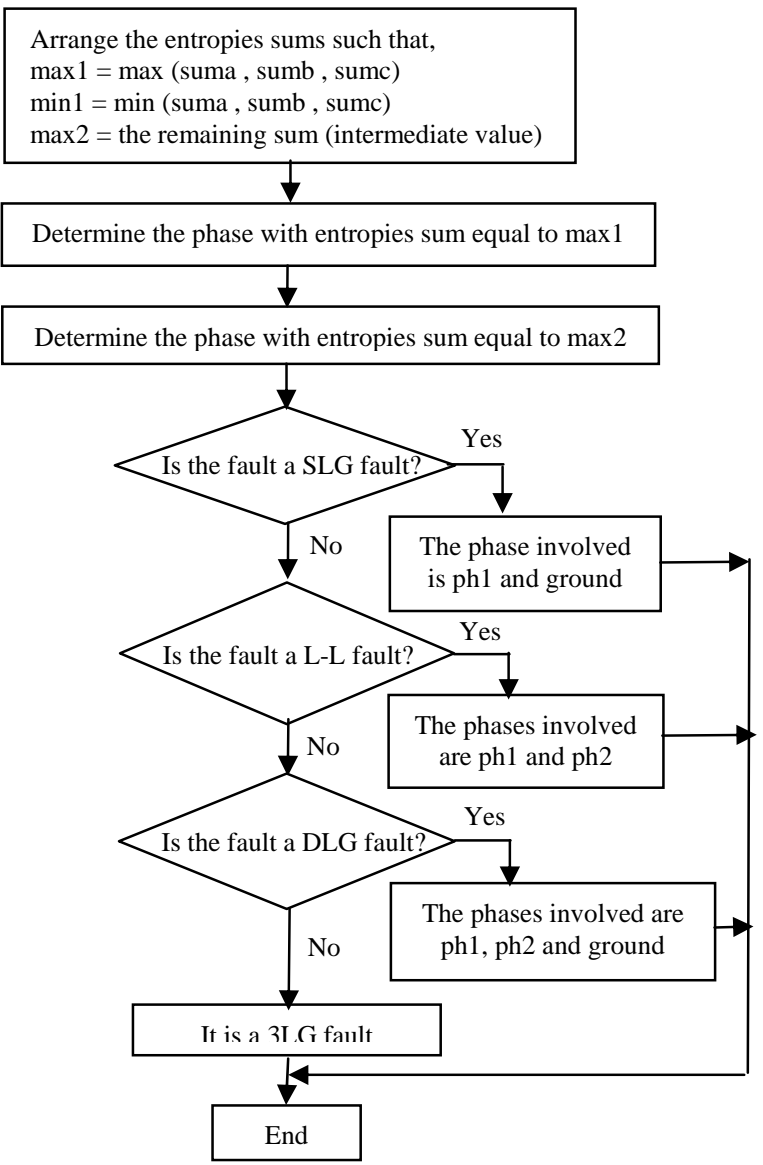

Fig. 2 Flow chart of phase selection algorithm

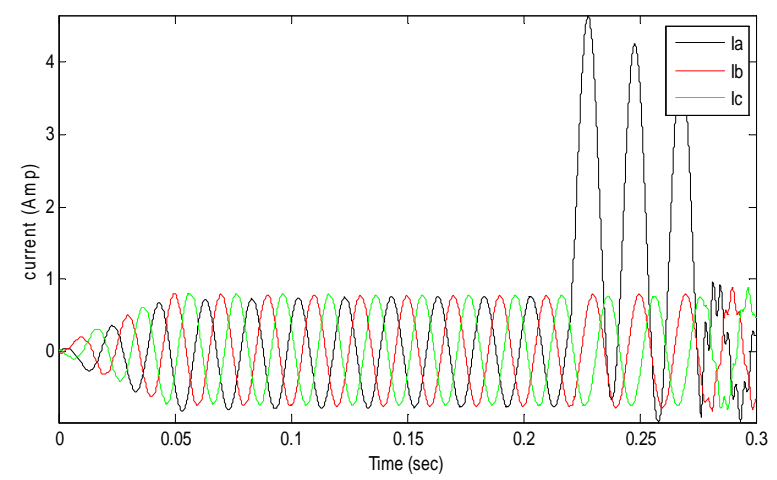

Fig. 3 Three phase currents for A-G fault 


\section{Test Results}

The current signals obtained from system simulation are opened with MATLAB, the ground current is calculated. Using the wavelet toolbox, the current signals are decomposed using 2 level Haar wavelet. Several wavelet families were examined and it was noticed that it makes no difference in the values of the entropies sums.

Fig. 4 shows the detailed and approximation of the line to ground fault obtained from the MATLAB wavelet toolbox.
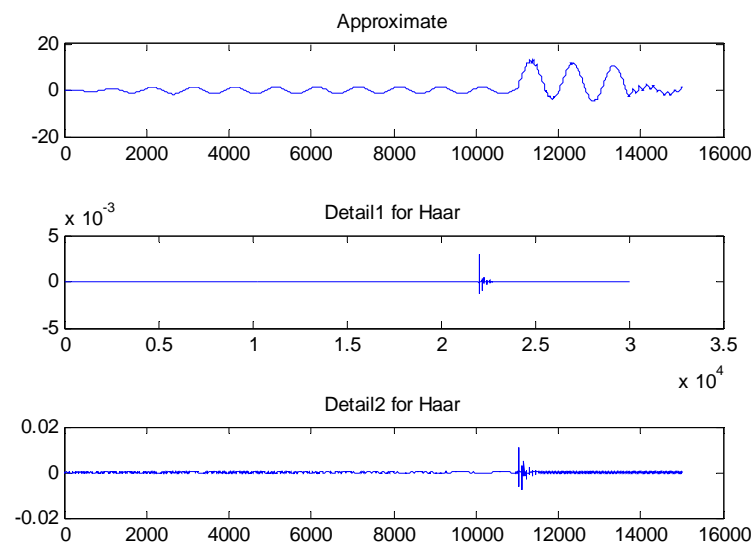

Fig. 4 Approximate and Details for phase A current

Table I shows the sums of wavelet coefficients entropies of the four currents.

TABLE I

The WAVElet Entropies Sums OF THE Three Phase AND Ground CURRENTS UNDER DIFFERENT SYSTEM CONDITIONS

\begin{tabular}{|c|c|c|c|c|}
\hline $\begin{array}{c}\text { Fault } \\
\text { Type }\end{array}$ & suma & sumb & sumc & sumg \\
\hline AB & $6.0354 \mathrm{e}+005$ & $4.4352 \mathrm{e}+005$ & $6.9734 \mathrm{e}+003$ & 0 \\
\hline BC & $6.8788 \mathrm{e}+003$ & $3.6524 \mathrm{e}+005$ & $2.1949 \mathrm{e}+005$ & 0 \\
\hline CA & $5.6659 \mathrm{e}+005$ & $6.8709 \mathrm{e}+003$ & $7.4036 \mathrm{e}+005$ & 0 \\
\hline AG & $3.0057 \mathrm{e}+005$ & $7.2072 \mathrm{e}+003$ & $6.9623 \mathrm{e}+003$ & $3.0357 \mathrm{e}+005$ \\
\hline BG & $6.9279 \mathrm{e}+003$ & $1.2962 \mathrm{e}+005$ & $7.3106 \mathrm{e}+003$ & $1.3231 \mathrm{e}+005$ \\
\hline CG & $7.2180 \mathrm{e}+003$ & $6.9223 \mathrm{e}+003$ & $1.9457 \mathrm{e}+005$ & $1.9764 \mathrm{e}+005$ \\
\hline ABG & $7.4752 \mathrm{e}+005$ & $3.7257 \mathrm{e}+005$ & $7.1938 \mathrm{e}+003$ & $7.7568 \mathrm{e}+004$ \\
\hline BCG & $7.1099 \mathrm{e}+003$ & $3.1790 \mathrm{e}+005$ & $2.7062 \mathrm{e}+005$ & $1.6280 \mathrm{e}+005$ \\
\hline CAG & $6.6925 \mathrm{e}+005$ & $7.4527 \mathrm{e}+003$ & $6.8548 \mathrm{e}+005$ & $5.7053 \mathrm{e}+004$ \\
\hline $\begin{array}{c}\text { No } \\
\text { Fault }\end{array}$ & $6.9072 \mathrm{e}+003$ & $6.8789 \mathrm{e}+003$ & $6.9634 \mathrm{e}+003$ & 0 \\
\hline
\end{tabular}

The proposed algorithm successfully detected and identified the fault at each case. As an example on how the algorithm works let us consider a line to line fault. As shown in Table I, the value of sumg in case of L-L fault and in case of no fault is equal to 0 but the entropies sums of the three phase currents in case of $\mathrm{L}-\mathrm{L}$ fault is much higher than that in case of no fault. That is how the threshold in the flow chart in Fig. 1 is determined. It is also noticed that in case of $A B$ fault, suma and sumb are higher than sumc. This way phases involved in the fault were selected.

\section{CONCLUSION}

The transient current waveform during the fault has been decomposed using wavelet transform. A novel technique composed of measuring the wavelet entropy of the decomposed waveforms was used for defining both the type of fault and the faulted phase. The proposed technique has been tested using different fault types and all the cases were successfully defined.

\section{REFERENCES}

[1] M. Kezunovic and I. Rikalo, "Detect and classify faults using neural nets,” IEEE Comput. Appl. Power, vol. 9, pp. 42-47, Oct. 1996.

[2] W. A. Wilkinson and M. D. Cox, "Discrete wavelet analysis of power system transients,” IEEE Trans. Power Syst., vol. 11, pp. 2038-2044.

[3] Bin Zhang, Zhengyou He and Qingquan Qian, "Application of wavelet entropy and adaptive nerve-fuzzy inference to fault classification”, Proc. Int. Conf. on Power System Technology, 2006.

[4] Zhengyou He, Yumei Cai and Qingquan Qian, “A study of wavelet entropy theory and its applications in power system”, Proc. of Int. Conf. on Intelligent Mechatronics and Automation, China, Aug. 2004.

[5] Zhimin Li, Weixing $\mathrm{Li}$ and Ruiye Liu, "Applications of entropy principles in power system: A survey", IEEE/PES Transmission and Distribution conference and Exhibition, China, 2005.

[6] H.Zheng-you, C.Xiaoqing and L.Guoming, "Wavelet Entropy Definition and its Application for Transmission Line Fault Detection and Identification (Part I: Definition and Methodology), Proc. Int. Conf. on Power System Technology, 2006.

[7] H.Zheng-you, C.Xiaoqing and L.Guoming, "Wavelet Entropy Definition and its Application for Transmission Line Fault Detection and Identification (Part III: Transmission line faults transients identification), Proc. Int. Conf. on Power System Technology, 2006.

[8] MATLAB reference manual, The Mathworks Inc., 2002. 